Gomariz Vicente, M.A., Martínez-Segura, M.J. \& Parra Martínez, J. (2019). Desde la implicación en el hogar de las familias a la facilitación de los docentes en un contexto multicultural. Revista Electrónica Interuniversitaria de Formación del Profesorado, 22(3), 45-60.

\title{
Desde la implicación en el hogar de las familias a la facilitación de los docentes en un contexto multicultural
}

\author{
María Ángeles Gomariz Vicente, María José Martínez-Segura, Joaquín Parra Martínez \\ Universidad de Murcia
}

\section{Resumen}

Familia y escuela deben permanecer unidas en la responsabilidad compartida de educar a niños y jóvenes. Desde este trabajo, nos planteamos conocer cuál es la implicación de las familias en la educación escolar desde el hogar, y en qué medida los docentes propician esta implicación. El estudio forma parte de una investigación (evaluativa y colaborativa) más amplia, con participación de 3506 familias y 225 docentes, de las etapas de Educación Infantil, Primaria y ESO, en un contexto socio cultural en el que prima la multiculturalidad. La información se ha obtenido a partir de cuestionarios (uno destinado a las familias y otro a los docentes), creados ad hoc, que contaban con siete dimensiones, de las cuales aquí sólo utilizamos los 14 ítems de la dimensión "Implicación de las familias en la educación escolar desde el hogar". Dichos instrumentos de recogida de información tuvieron en cuenta las necesidades idiomáticas de las familias (español, árabe e inglés) y de los docentes (español). La fiabilidad de los instrumentos fue alta (Alfa de Cronbach de 0,876 y 0,982). El análisis de resultados pone de manifiesto que las familias valoran muy positivamente su implicación desde el hogar y, al mismo tiempo, matizados siempre por el propio contexto multicultural, los docentes se muestran facilitadores de la mencionada implicación. Se destaca la afición por la lectura y la promoción de actividades culturales como las acciones mejorables.

\section{Palabras clave}

Familia; docentes; contexto multicultural; enseñanza obligatoria.

\section{Contacto:}

Joaquín Parra Martínez, jparra@um.es

Proyecto I+D+I EDU2016-77035-R Compartimos la educación. Programa para el fomento de la participación de las familias en los centros educativos 


\title{
From the involvement at home of the families to the facilitation of teachers in a multicultural context
}

\begin{abstract}
Family and school must stand together in the shared responsibility of educating children and young people. From this work, we ask to know what is the involvement of families in education from home, and what teachers promote this involvement. The study, is part of a broader research (evaluative and collaborative), with the participation of 3506 families and 225 teachers have participated, of the Educational stages of Preschool, Primary and Secondary, in a multicultural context. The information was obtained from questionnaires (one for families and one to teachers), created ad hoc, with seven dimensions, and here only use 14 items in the dimension "Family involvement in education from home". These instruments for collecting information were considered the language needs of families (Spanish, Arabic and English) and teachers (Spanish). The reliability of the instruments was high (Cronbach's alpha of 0.876 and 0.982). The analysis of results shows that families valued very positively its involvement from the home and, at the same time, teachers are facilitators of mentioned involvement. It highlights the hobby of reading and the promotion of cultural activities such as improved actions.
\end{abstract}

\section{Key words}

Family; teachers; multicultural context; compulsory education.

\section{Introducción}

En el proceso educativo de nuestros niños y jóvenes es indiscutible hoy día que familia y escuela han de compartir responsabilidad y objetivos educativos comunes, creando relaciones basadas en el respeto mutuo, la consideración del otro, las relaciones de comunicación positiva y la reciprocidad (Bolívar, 2006; Epstein, 2009; Forest y García Bacete, 2006). Para que esto suceda, familia y escuela deben compartir objetivos, tiempos, espacios y estrategias.

Son numerosas las investigaciones que evidencian la influencia de la participación educativa de las familias en la mejora del proceso de aprendizaje de sus hijos, en la motivación de los mismos, en su rendimiento académico y en el éxito escolar (González-Pineda, 2003; Fernández-Hawrylak y Heras, 2019; Santos, Ferraces, Godas y Lorenzo, 2018; Epstein y Sheldon, 2002).

La participación de las familias en los centros educativos es un fenómeno multidimensional, tal y como muestran diversos estudios de carácter meta-analítico (Castro, Expósito, Lizasoain, López y Navarro, 2014; Chen y Gregory, 2010; Fan y Chen, 2001). Por ello, tiene múltiples formas de manifestarse, en unas se refiere a las relaciones de comunicación entre familias y escuela, en otras a las acciones de participación en los propios centros educativos (a nivel de aula, centro o comunidad). En relación a la participación, se materializa a través de órganos formales como AMPA y CE, y en otras a acciones de apoyo y acompañamiento o contribución a los aprendizajes escolares desde el hogar. En este último aspecto, se centra nuestra atención. Si nos situamos desde la perspectiva del propio contexto familiar, podríamos hablar de la dimensión desarrollo de los aprendizajes (Parra Martínez, SánchezLópez y Gomariz Vicente, 2011). Desde la perspectiva del estudio de la participación de las familias en la vida de los centros, nos referimos a la implicación familiar desde el hogar en el 
proceso educativo de los hijos, o lo que Hernández-Prados, Gomariz Vicente, Parra Martínez, y García-Sanz (2016) denominan implicación directa.

Encontramos autores que consideran el estudio de manera diferenciada de la implicación de las familias; en el contexto escolar, por una parte, y en el contexto del hogar, por la otra (Anderson y Minke, 2007; Hoover-Dempsey et al., 2005). Sin embargo, nuestro modelo de acercamiento al fenómeno de participación de las familias en la educación, opta por incluir la implicación de los padres y madres en los aprendizajes escolares desde el hogar, como una de las dimensiones relevantes en dicha participación. Así, coincidimos con Epstein (2009) cuando habla de aprendizaje en el hogar, para referirse a la participación de las familias en la que se les puede facilitar información para que ayuden a sus hijos con las tareas escolares en casa, así como con todas aquellas cuestiones y decisiones de carácter académico. Sin embargo, consideramos que para las familias, en contraste con los docentes, esta implicación va más allá de lo meramente académico, se extiende hasta proporcionar a su hijo seguridad, salud, bienestar, y en ese apoyo, intentar alcanzar el éxito académico, escolar y social (Etxeberria e Intxausti, 2013; Lawson, 2003). Se trataría por tanto, del espacio de participación de las familias en la educación de sus hijos que hace referencia a la esfera propiamente intrafamiliar, y que tal y como defienden Parra Martínez, Gomariz Vicente, Hernández-Prados y García-Sanz, (2017) alude a la responsabilidad y funciones ineludibles de la familia: cuidado, protección y educación; reconocidas social y culturalmente, de manera más intensa, desde la Declaración Universal de los Derechos del Niño (1959).

Respecto al estudio de esta dimensión desde la perspectiva del profesorado, las investigaciones suelen centrarse en la percepción que tienen los docentes acerca de la implicación de las familias desde el hogar, y no son coincidentes los hallazgos obtenidos. Mientras que casi la mitad del profesorado (46,3\%) manifiesta que las familias no atienden suficientemente las tareas escolares de sus hijos (Martín, Rodríguez y Marchesi, 2005); otros estudios como el de Madrona (2001), revelan que una amplia mayoría de los docentes (83\%) afirma que las familias colaboran en las actividades escolares de sus hijos desde el hogar.

Nuestro modelo adopta una perspectiva diferente: nos situamos desde la premisa de la corresponsabilidad entre familia y escuela, es decir, consideramos que el docente es el agente que ha de facilitar esta implicación familiar. Si atendemos a la normativa educativa, en la propia Ley Orgánica de Educación (LOE, 2006) encontramos, entre las funciones del profesorado, al menos dos que dirigen la atención sobre las familias del alumnado:

c) La tutoría de los alumnos, la dirección y la orientación de su aprendizaje y el apoyo en su proceso educativo, en colaboración con las familias.

h) La información periódica a las familias sobre el proceso de aprendizaje de sus hijos e hijas, así como la orientación para su cooperación en el mismo (art. 91.c, 91.h).

Los docentes suelen establecer relaciones de causa-efecto entre no asistir o no participar en actividades del centro, y niveles de escasa implicación desde el hogar. Nosotros defendemos, al igual que Andrés y Giró (2016) que los centros y los docentes, en especial los tutores (Etxeberria e Intxausti, 2013) han de convertirse en los principales facilitadores de la implicación de las familias en los aprendizajes desde el hogar. De este modo, los progenitores, independientemente de su nivel educativo, se involucran de manera más intensa cuando los docentes lo promueven activamente (Dauber y Epstein, 1989). Y esta facilitación se puede dar a través de acciones concretas que las familias pueden desarrollar y los docentes deben estimular. Por una parte, nos referimos a acciones que no implican que las familias tengan que dominar las materias curriculares, sino mostrar interés por las tareas escolares, comunicarse con el hijo sobre la vida escolar, felicitar al hijo por los logros y éxitos 
alcanzados o mostrar explícitamente su valoración por la educación (Bonell, 2016; HooverDempsey et al., 2001). Por otra parte, de acuerdo con Grant y Ray (2013), las familias pueden desarrollar estrategias de implicación que requieren de ciertos conocimientos sobre el proceso de desarrollo de sus hijos, los procesos de aprendizaje o las materias curriculares. Es en estas últimas acciones, en las que requerirían de una mayor orientación por parte del centro educativo y su profesorado, lo que incide en la relevancia de que se promuevan verdaderas relaciones de colaboración entre la escuela y todas y cada una de sus familias (Álvarez Blanco, 2019). Otra de las estrategias que se proponen para favorecer esta implicación, especialmente en las familias de origen no español, es partir de su potencial educativo y bagaje cultural (Flecha y Soler, 2013; López, Scribner y Mahitivanichcha, 2001), promoviendo actuaciones que atiendan sus necesidades de formación (Bonell, 2016) así como partiendo de la premisa que todas las familias, independientemente de su origen o nivel educativo, pueden ofrecer apoyo positivo en los aprendizajes de sus hijos (Valls-Caroll, Prados-Gallardo y Aguilera-Jiménez, 2014).

Si bien es cierto que el grado de participación de las familias en la educación disminuye conforme el niño avanza en la escolaridad (Garreta, 2008), también se demuestra más necesaria en la etapa educativa de Secundaria, frente a etapas educativas tempranas, en donde se relaciona más intensa y significativamente con el rendimiento escolar (Castro et al., 2014). Parece que las familias concentran sus esfuerzos para participar, y los centros para atraerlas, en los primeros niveles de escolaridad, cuando su labor crucial de acompañamiento se hace más precisa en niveles educativos avanzados. La implicación parental desde el hogar en el proceso educativo de los hijos, se ha de dar desde el comienzo de la escolaridad (Fernández-Freire, Rodríguez-Ruiz y Martínez-González, 2019; García, 2016) e irá evolucionando con el avance del proceso evolutivo y escolar del niño (Parra et al., 2017).

De acuerdo con lo expuesto, nos planteamos dos objetivos en este trabajo:

1. Conocer la implicación de las familias del alumnado en la educación escolar desde el hogar y su correspondencia con la facilitación de esa implicación por parte del profesorado.

2. Analizar la implicación en el hogar de las familias y la facilitación del profesorado respecto a ella en función variables sociodemográficas relevantes (origen nacional, titularidad del centro, etapa educativa, situación laboral del docente, experiencia docente).

\section{Metodología}

Los resultados aquí expuestos constituyen un estudio de corte transversal basados en parte de los datos de una encuesta aplicada a familias y docentes. Se trata de una investigación más amplia derivada del proyecto I+D+I que se referencia que no podría exponerse en el número de palabras que requiere habitualmente una revista científica. Más allá de una explotación de los datos obtenidos de manera parcelada, por secciones como quien usa un micrótomo, supone ahondar en uno de los aspectos clave de la participación de las familias en los centros escolares según el modelo teórico en el que se sustenta el trabajo. Así será posible extraer conclusiones que contribuyan a la mejora de dicha implicación. 


\section{Participantes}

El contexto elegido para realizar esta investigación presenta una característica que es muy particular y es donde reside uno de sus principales valores como investigación: la multiculturalidad, un contexto crítico, donde las relaciones entre familia y escuela encuentran mayores dificultades para surgir espontáneamente. Las posibles inferencias sobre la generalización de sus resultados han de considerarse siempre desde esta valiosa circunstancia.

El presente trabajo se ha desarrollado en un municipio de la Región de Murcia (España). Cerca del $70 \%$ de los 35.000 habitantes son de nacionalidad española, mientras que cerca del $30 \%$ lo es de nacionalidad no española (en torno al $18 \%$ del total proceden de África, el $5 \%$ de América, siendo del resto Europa también el $5 \%$, y un $2 \%$ de Asia). El sector principal de actividad es la agricultura intensiva. Se ha contado con dos tipos de informantes: familias y docentes. Familias cuyos hijos e hijas cursaban desde Educación Infantil (tres años) hasta cuarto de Educación Secundaria Obligatoria (ESO); y profesorado que desempeñaba su labor docente en estos mismos niveles educativos. Se partió de una muestra inicial de 3639 familias que propiciaron una muestra real válida contestando a esta dimensión en concreto 3506 familias, de una población estimada de 5022 familias. Junto a ellas se contó con 225 docentes de una población de 718. Las muestras participantes pertenecían a 14 centros (la totalidad de la población de centros del municipio), de los cuales, dos de ellos son privado-concertados, con enseñanza en las tres etapas educativas; nueve son públicos, uno de ellos con las etapas de Educación Infantil hasta Secundaria Obligatoria, y ocho con enseñanza en Educación Infantil y Primaria; y tres son públicos, con solo Educación Secundaria (Tablas 1, 2 y 3).

Tabla 1.

Origen nacional de las familias. Porcentajes válidos

\begin{tabular}{ll}
\hline Origen nacional & $\%$ \\
\hline Español padres y madres & 59,2 \\
Padres o madres no españoles & 40,8 \\
\hline
\end{tabular}

Tabla 2.

Titularidad del centro. Porcentajes válidos

\begin{tabular}{ll}
\hline Tipo de centro & $\%$ \\
\hline Público & 82,0 \\
Privado concertado & 18,0 \\
\hline
\end{tabular}

Tabla 3.

Etapa educativa. Porcentajes válidos

\begin{tabular}{lc}
\hline Etapa educativa & $\%$ \\
\hline Educación Infantil & 22,5 \\
Educación Primaria & 58,5 \\
Educación Secundaria Obligatoria & 18,6 \\
\hline
\end{tabular}




\section{Diseño}

El presente trabajo se configura como un estudio cuantitativo, no experimental, exploratorio y de carácter evaluativo. Desde el modelo de partida basado metodológicamente en GarcíaSanz (2012), supone la detección de necesidades mostradas por las familias y la manera de dar respuesta a la participación como agentes facilitadores de la participación por parte del profesorado.

\section{Instrumentos}

Para alcanzar los objetivos previstos se ha partido de la aplicación de dos cuestionarios, uno dirigido a las familias titulado "Valoración de la participación de las familias en la educación de los hijos (Hernández-Prados, Gomariz Vicente, Parra Martínez y García-Sanz, 2017) y otro dirigido al profesorado denominado "Facilitación de la participación de las familias en la educación de los hijos" (Gomariz Vicente, Hernández-Prados, García-Sanz y Parra Martínez, 2017). Para el presente trabajo se ha utilizado la dimensión Implicación de las familias en la educación escolar desde el hogar, que está compuesta por un total de quince ítems en cada una de sus versiones (familias y profesorado). Ambos, se pueden cotejar íntegros en el repositorio de la Universidad de Murcia , pues están alojados según las referencias.

Para trabajar la dimensión (Implicación de las familias en la educación escolar desde el hogar), hemos utilizado sendas partes específicas de los mencionados cuestionarios. Se puede apreciar en detalle más adelante en las Tablas 4 y 5 . Se trata de 14 ítems coincidentes en ambos casos en los que, según el modelo teórico de partida de los autores, se recoge la realidad de la implicación (para las familias) y cómo se facilita la implicación por parte del profesorado. La escala de respuesta, tipo Lickert, es para todos los ítems de cinco valores: 1, Nunca/nada; 2: Pocas veces/poco; 3: Algunas veces/algo; 4: Frecuentemente/bastante; 5: Siempre/mucho.

A propósito de la calidad de los instrumentos, ambos cuestionarios fueron sometidos a validación por expertos (cinco profesores universitarios con formación específica en el contenido de los instrumentos), por equipos directivos de los centros escolares, así como por las directivas de las Asociaciones de Madres y Padres de Alumnos (AMPA).

La fiabilidad de los cuestionarios fue muy alta, arrojaron un Alfa de Cronbach de 0,966 en el cuestionario dirigido a las familias y de 0.981 en el cuestionario dirigido al profesorado. En el caso concreto de la dimensión que nos ocupa, Implicación de las familias en la educación escolar desde el hogar, el Alfa de Cronbach en el caso de los ítems dirigidos a las familias resultó ser de 0,876 , mientras que respecto al caso de los ítems dirigidos al profesorado fue de 0,928 .

Para el cuestionario de familias, en el intento de reducir la dimensión, hemos desarrollado el análisis factorial pertinente y en el test de esfericidad de Bartlett hemos encontrado que existe correlación con $\mathrm{p}<.000$, siendo el índice KMO regular $(0,928)$. En el conjunto de la dimensión aparecen dos componentes que saturan el 50,749 del porcentaje de la varianza. En el primero de ellos se recogen todos los ítems relacionados con la comunicación, apoyo y seguimiento en el aprendizaje de los hijos e hijas. En el segundo componente se incorpora ítems acerca de las características de la familia como lectora o asidua visitante de museos, actividades culturales, etc.; no son acciones de implicación sino características o funcionamiento (hábitos) de la familia que se supone influye la educación de los hijos como 
modelaje: leer, visitar museos, etc. Se puede inferir por tanto que la dimensión considerada aparece como un constructo de contenido sólido en función de lo que se pretende medir.

En el caso del cuestionario a profesores, respecto a la dimensión que nos ocupa, en el test de esfericidad de Bartlett hemos encontrado que existe correlación con $p<.000$, siendo el índice KMO regular $(0,922)$. En este caso, cuestionario a los docentes, aparecen dos componentes que acumulan el $61,785 \%$ de la varianza. Supone un constructo coherente en cuanto a contenido. El primero de ellos presenta contenido referido al apoyo y seguimiento en el aprendizaje, mientras que el segundo lo hace respecto a la creación de un clima de confianza comunicación de la familia con su hijos e hijas que permita la comunicación, así como la transferencia de lo aprendido en el aula a la vida cotidiana.

\section{Procedimiento}

Los cuestionarios fueron facilitados, aula por aula, a cada tutor según los posibles requerimientos idiomáticos de las propias familias (español, árabe o inglés). Las respuestas al cuestionario dirigido a profesores se dieron a través de la plataforma de "Encuestas" de la nuestra universidad, donde se alojó el instrumento. Con posterioridad, se recogieron por parte de los investigadores centro a centro todos los cuestionarios y se creó la base de datos correspondiente.

\section{Análisis de datos}

Para la elección de las técnicas a utilizar en el análisis de datos se aplicó la prueba de Levene de homocedasticidad y prueba de normalidad de Kolmogorov-Smirnov, ofreciendo valores que nos llevaron a la consideración de técnicas no paramétricas según los objetivos. Así, se han utilizado las pruebas U Mann-Whitney y KrusKal-Wallis, así como el cálculo del tamaño del efecto. El análisis de datos se ha realizado usando el paquete estadístico SPSS v.24.

\section{Resultados}

Para la presentación de resultados de la investigación, partiremos de cada uno de los objetivos inicialmente formulados.

Objetivo 1. Conocer la implicación de las familias del alumnado en la educación escolar desde el hogar y su correspondencia con la facilitación de esa implicación por parte del profesorado.

En primer lugar, para dar cuenta del primer objetivo general podemos considera y apreciar la forma de darse las medias de los ítems que componen y con la que hemos definido el constructo implicación de las familias en la educación escolar desde el hogar (Tabla 4). La media global de la dimensión es alta $(4,16)$, por lo que las familias valoran muy positivamente, de forma general, su implicación en la educación escolar desde el hogar, lo que en un rango de variabilidad de 1 a 5 resulta un valor a ponderar. Mucho más relevante es, incluso, cuando manifiestan que están muy informados de la asistencia de sus hijos e hijas a clase $(4,60)$, expresan que se interesan en gran medida por las tareas que han de realizar en casa $(4,59)$, reforzando a su hijo positivamente cuando realiza las tareas $(4,51)$, potenciando un buen clima de estudio en casa $(4,47)$, a la vez que se preocupan por la organización de su tiempo de estudio $(4,47)$. En cuanto a la actitud general de las familias ante el estudio de los hijos, se expresa con disposición de ayuda en las tareas escolares $(4,45)$, comunicativas respecto a lo que ocurre en las aulas $(4,32)$ y de mostrar confianza en los hijos $(4,43)$. Son valores sobre los que reflexionar dadas sus dimensiones. Las medias más bajas, siempre por encima de la 
puntuación 3, se corresponden con los ítems que reflejan características de las familias y desde las que supuestamente se puede inferir cierta función de modelaje como son los ítems que hacen referencia a los hábitos lectores de las familias $(3,14)$, así como la asistencia al cine, teatro, museos, viajes, conciertos, exposiciones, etc. $(3,13)$. Resulta importante subrayar que el altísimo porcentaje de personas migrantes que no saben leer y escribir en su idioma materno, así como los contextos interculturales de origen explican esta media de respuesta al ítem. Lo mismo sucede con el ítem referido a la facilitación de actividades extracurriculares o complementarias por parte de las familias, ya que existe un porcentaje muy considerable que por razones económicas no se lo pueden permitir.

Tabla 4.

Porcentajes y medias de los ítems sobre Implicación de las familias en la educación escolar desde el hogar

\begin{tabular}{|c|c|c|c|c|c|c|}
\hline \multirow{3}{*}{ Ítems } & \multicolumn{6}{|c|}{ Familias } \\
\hline & \multicolumn{5}{|c|}{ Opciones de respuesta. Porcentajes } & \multirow{2}{*}{ Media* } \\
\hline & Nada & Poco & Algo & Bastante & Mucho & \\
\hline $\begin{array}{l}\text { Conversación con el hijo sobre lo que se ha } \\
\text { tratado en el aula. }\end{array}$ & 1,1 & 2,9 & 10,7 & 33,0 & 52,3 & 4,32 \\
\hline $\begin{array}{l}\text { Manifestación al hijo de la confianza que se } \\
\text { tiene en él. }\end{array}$ & 0,8 & 2,9 & 7,9 & 28,6 & 57,9 & 4,43 \\
\hline $\begin{array}{l}\text { Información sobre la asistencia del hijo a } \\
\text { clase. }\end{array}$ & 3,3 & 1,3 & 3,8 & 14,8 & 76,7 & 4,60 \\
\hline $\begin{array}{l}\text { Interés por las tareas escolares a realizar en } \\
\text { casa. }\end{array}$ & 1,3 & 1,7 & 5,1 & 20,3 & 71,5 & 4,59 \\
\hline $\begin{array}{l}\text { Preocupación por cómo organiza el tiempo } \\
\text { de estudio. }\end{array}$ & 0,9 & 2,3 & 6,8 & 29,0 & 60,9 & 4,47 \\
\hline $\begin{array}{l}\text { Potenciación del buen clima de estudio en } \\
\text { casa. }\end{array}$ & 1,2 & 1,5 & 7,7 & 28,6 & 61,0 & 4,47 \\
\hline $\begin{array}{l}\text { Disponibilidad para ayudar con las tareas } \\
\text { escolares. }\end{array}$ & 1,4 & 3,2 & 8,5 & 23,0 & 63,8 & 4,45 \\
\hline $\begin{array}{l}\text { Felicitación al hijo cuando realiza sus tareas } \\
\text { escolares. }\end{array}$ & 1,8 & 2,3 & 6,5 & 21,6 & 67,7 & 4,51 \\
\hline $\begin{array}{l}\text { Facilitación de actividades extracurriculares } \\
\text { o complementarias. }\end{array}$ & 10,2 & 9,6 & 17,3 & 20,7 & 42,2 & 3,75 \\
\hline $\begin{array}{l}\text { Fomento de la autonomía y responsabilidad } \\
\text { en el estudio. }\end{array}$ & 2,3 & 4,7 & 12,0 & 29,0 & 51,9 & 4,24 \\
\hline $\begin{array}{l}\text { Control en el uso responsable de } \\
\text { ordenadores, móviles. }\end{array}$ & 2,9 & 4,3 & 8,8 & 27,5 & 56,4 & 4,30 \\
\hline Afición hacia la lectura. & 7,3 & 21,7 & 34,7 & 22,5 & 13,8 & 3,14 \\
\hline $\begin{array}{l}\text { Asistencia al cine, teatro, museos, viajes, } \\
\text { conciertos, exposiciones, etc. }\end{array}$ & 11,1 & 18,8 & 32,2 & 22,0 & 15,9 & 3,13 \\
\hline $\begin{array}{l}\text { Ayuda para la aplicabilidad en la vida } \\
\text { cotidiana de lo aprendido en clase. }\end{array}$ & 1,4 & 5,4 & 16,7 & 39,3 & 37,3 & 4,06 \\
\hline
\end{tabular}




\begin{tabular}{|c|c|c|c|c|c|}
\hline \multirow{3}{*}{ Ítems } & \multicolumn{5}{|c|}{ Familias } \\
\hline & \multicolumn{4}{|c|}{ Opciones de respuesta. Porcentajes } & \multirow{2}{*}{ Media* } \\
\hline & Nada Poco & Algo & Bastante & Mucho & \\
\hline Media Global Dimensión & & & & & 4,16 \\
\hline $\begin{array}{l}\text { El centro promueve la implicación de la fami } \\
\text { hijos }\end{array}$ & a desde el hoga & en los & aprendizaje & de los & 3,94 \\
\hline
\end{tabular}

*Rango de variabilidad de 1 a 5 .

Cuando consideramos la percepción que las familias tienen respecto a las facilidades que da el centro para que se impliquen desde el hogar, se encuentra una correlación positiva (Rho de Spearman de 0,454; $p<, 000)$ entre la media global de la dimensión $(\bar{x}=4,16)$ con la referida a la mencionada facilitación $(\bar{x}=3,94)$. Así, cabe pensar que a mayor facilitación por parte del centro más alta es la implicación desde el hogar (Tabla 4).

Al contemplar las percepciones del profesorado, se puede apreciar que no ha existido por parte de las familias de la muestra tendencia a la deseabilidad social o la manipulación de la propia imagen, difícil de sostener teniendo en cuenta el tamaño de la muestra. Esa percepción de las familias con respecto al comportamiento del profesorado coincide con la propia consideración que los docentes tienen al respecto (Tabla 5). En este caso, el profesorado percibe, curiosamente, con medias superiores al valor 4 (Frecuentemente/bastante), prácticamente todos los ítems, a excepción del referido a su facilitación de información a las familias sobre la importancia de que los hijos realicen actividades extracurriculares o complementarias que se sitúa en 3,92, y el relacionado con el fomento de la asistencia de las familias a actividades culturales (cine, teatro, museos, conciertos, exposiciones, ... ) que presenta la puntuación media más baja $(3,86)$, aún siendo superior al valor crítico de 3. Respecto a las puntuaciones medias más elevadas, encontramos que el ítem más valorado por el profesorado participante coincide con proporcionar de manera frecuente a las familias información sobre la asistencia de su hijo a clase $(4,54)$.

Tabla 5.

Porcentajes y medias de los ítems sobre la facilitación del profesorado de la implicación de las familias en la educación escolar desde el hogar

\begin{tabular}{lccccccc}
\hline \multirow{2}{*}{\multicolumn{1}{c}{ Ítems }} & \multicolumn{6}{c}{ Docentes } \\
\cline { 2 - 6 } & \multicolumn{5}{c}{ Opciones de respuesta. Porcentajes } & Media* \\
\cline { 2 - 6 } & Nada & Poco & Algo & Bastante & Mucho & \\
\hline $\begin{array}{l}\text { Oriento a los padres para que hablen con su } \\
\text { hijo sobre lo que se ha tratado en el aula. }\end{array}$ & 0,9 & 3,6 & 15,1 & 35,6 & 44,9 & 4,20 \\
\hline $\begin{array}{l}\text { Animo a las familias a que expresen a su } \\
\text { hijo que confían en él. }\end{array}$ & 0,9 & 3,6 & 10,2 & 32,4 & 52,9 & 4,33 \\
\hline $\begin{array}{l}\text { Informo a las familias sobre la asistencia del } \\
\text { hijo a clase. }\end{array}$ & 1,3 & 1,8 & 6,2 & 23,1 & 67,6 & 4,54 \\
\hline $\begin{array}{l}\text { Promoción del interés de las familias por las } \\
\text { tareas escolares a realizar en casa. }\end{array}$ & 0 & 1,3 & 6,2 & 33,3 & 59,1 & 4,50 \\
\hline $\begin{array}{l}\text { Preocupación por cómo organiza el tiempo } \\
\text { de estudio. }\end{array}$ & 0 & 1,3 & 5,3 & 34,7 & 58,7 & 4,51 \\
\hline $\begin{array}{l}\text { Potenciación del buen clima de estudio en } \\
\text { casa. }\end{array}$ & 0,4 & 0,9 & 5,8 & 33,8 & 59,1 & 4,50 \\
\hline
\end{tabular}




\begin{tabular}{|c|c|c|c|c|c|c|}
\hline \multirow{3}{*}{ Ítems } & \multicolumn{6}{|c|}{ Docentes } \\
\hline & \multicolumn{5}{|c|}{ Opciones de respuesta. Porcentajes } & \multirow{2}{*}{ Media* } \\
\hline & Nada & Poco & Algo & Bastante & Mucho & \\
\hline $\begin{array}{l}\text { Disponibilidad para ayudar con las tareas } \\
\text { escolares. }\end{array}$ & 0,4 & 2,7 & 8,0 & 41,3 & 47,6 & 4,33 \\
\hline $\begin{array}{l}\text { Felicitación al hijo cuando realiza sus tareas } \\
\text { escolares. }\end{array}$ & 0,4 & 1,3 & 8,4 & 30,2 & 59,6 & 4,47 \\
\hline $\begin{array}{l}\text { Informo a los padres sobre la importancia } \\
\text { de que sus hijos realicen actividades } \\
\text { extracurriculares o complementarias. }\end{array}$ & 3,1 & 7,1 & 18,7 & 37,3 & 33,8 & 3,92 \\
\hline $\begin{array}{l}\text { Converso con las familias para su fomento } \\
\text { de la autonomía y responsabilidad en el } \\
\text { estudio. }\end{array}$ & 0 & 1,3 & 7,1 & 32,9 & 58,7 & 4,49 \\
\hline $\begin{array}{l}\text { Control parental en el uso responsable de } \\
\text { ordenadores, móviles. }\end{array}$ & 1,3 & 4,4 & 12,0 & 27,6 & 54,7 & 4,30 \\
\hline $\begin{array}{l}\text { Promuevo que las familias desarrollen } \\
\text { afición por la lectura. }\end{array}$ & 0,9 & 3,1 & 6,7 & 30,7 & 58,7 & 4,43 \\
\hline $\begin{array}{l}\text { Fomento en las familias que asistan al cine, } \\
\text { teatro, museos, viajes, conciertos, } \\
\text { exposiciones, etc. }\end{array}$ & 4,4 & 4,9 & 22,2 & 36,9 & 31,6 & 3,86 \\
\hline $\begin{array}{l}\text { Ayuda para la aplicabilidad en la vida } \\
\text { cotidiana de lo aprendido en clase. }\end{array}$ & 0,9 & 5,3 & 14,2 & 35,1 & 44,4 & 4,17 \\
\hline Media Global Dimensión & & & & & & 4,32 \\
\hline $\begin{array}{l}\text { El centro facilita la implicación de las familia } \\
\text { de sus hijos }\end{array}$ & desde e & hogar & n el pr & ceso educa & tivo & 4,32 \\
\hline
\end{tabular}

Al contemplar la relación que existe entre la percepción que tiene el profesorado de sí mismo como facilitadores de la implicación familiar en el hogar con la percepción que tienen esos mismos docentes respecto a lo que se hace en sus centros, se observa un coeficiente de correlación de Spearman positivo relevante $(0,669 ; \mathrm{p}<, 000)$. Digamos, pues, que el profesorado se suele comportar de manera homogénea o regular en los centros con respecto a la facilitación de la implicación de las familias en el hogar, por lo que no cabe pensar en deseabilidad social o manipulación de la propia imagen a la hora de responder.

Al comparar las medias de las contestaciones de familias y docentes en aquellos ítems homólogos (sabiendo que en el caso de las familias el contenido refleja cómo actúan las figuras parentales o progenitores y que en el caso de los docentes en qué medida facilitan, promueven, orientan, etc.), se aprecian diferencias estadísticamente significativas a favor de las contestaciones del profesorado, con un valor d de Cohen (1988) superior a 0,5, en los contenidos siguientes: "afición hacia la lectura" y, "asistencia al cine, teatro, museos, viajes, conciertos, exposiciones, etc." (1,309 y 0,641, respectivamente). Es decir, en opinión de los propios docentes, ellos facilitan, estimulan y promueven significativamente más de lo que las familias consideran que llevan a cabo su implicación respecto a estas dos acciones: la afición a la lectura y la asistencia a actividades de tipo cultural. Viene a ser un resultado esperado, teniendo en cuenta los niveles socioeconómicos y, sobre todo, culturales de una población 
en un contexto multicultural y rural como el que se define con la muestra participante, como se informa desde el propio título de este trabajo.

Objetivo 2. Analizar la implicación en el hogar de las familias y la facilitación del profesorado respecto a esa implicación en función variables sociodemográficas relevantes (origen nacional, titularidad del centro, etapa educativa, situación laboral del docente, experiencia docente).

Partiendo de un número total de 3506 de familias informantes (válidos respecto a esta dimensión de la participación que nos ocupa), es importante subrayar que el 58,5\% de las familias presentan figuras parentales de origen español, siendo el 40,4\% las familias en las que alguna o todas sus figuras parentales son de origen diferente al español. Tras aplica la Prueba $U$ de Mann-Whitney $(p<, 000$ ), podemos decir que existe diferencia significativa entre las medias (Tabla 6 ) obtenidas respecto a esta implicación desde el hogar entre las primeras de ellas ( $\bar{x}=4,33$; $D T=0,524)$ y aquellas familias en las que alguna al menos de las figuras paternas no son de origen español $(\bar{x}=3,93$; $D T=0,633)$, siendo el tamaño del efecto considerable ( $d$ de Cohen 0,688 , superior a $d=0,5$ ).

Tabla 6.

Origen nacional de las familias, titularidad de centro y etapa educativa. Medias y desviaciones típicas

\begin{tabular}{lcc}
\hline Variable sociodemográfica & Media & DT \\
\hline Origen nacional & & \\
\hline Español padre y madre & 4,33 & 0,524 \\
No español padre o madre & 3,93 & 0,633 \\
\hline Tipo de centro & & \\
\hline Público & 4,14 & 0,610 \\
Privado concertado & 4,33 & 0,513 \\
\hline Etapa educativa & & \\
\hline Educación Infantil & 4,17 & 0,577 \\
Educación Primaria & 4,22 & 0,561 \\
Educación Secundaria Obligatoria & 4,01 & 0,705 \\
\hline
\end{tabular}

Al considerar la titularidad del centro donde las familias escolarizan a sus hijos e hijas, debemos indicar que en el caso de las familias informantes el $82,0 \%$ de sus hijos o hijas lo hacen en centros públicos, mientras que el $18,0 \%$ lo hacen en centros concertados (laicos, de tipo de gestión cooperativa). Con los resultados del análisis podemos señalar que existe diferencia significativa $(\mathrm{p}<, 000)$ entre la media de las respuesta al conjunto de la dimensión respecto a las familias de centros concertados $(\bar{x}=4,33$; DT $=0,513)$ y las de quienes escolarizan a sus hijos o hijas en centros públicos ( $\bar{x}=4,14 ; \mathrm{DT}=0,610)$, aunque el pequeño tamaño del efecto no nos permite, y lo enfatizamos, considerar esta diferencia ( $d$ de Cohen 0,337 , inferior a d $=0,5)$. 
Existen diferencias significativas cuando reparamos en la etapa educativa, aun cuando solo se dan al comparar la forma de implicarse en el hogar las familias cuando sus hijos o hijas se encuentran cursando Educación Secundaria Obligatoria a cuando se encuentran cursando Educación Infantil o Primaria. No existen estas diferencias al comparar Infantil con Primaria $(\mathrm{p}=, 054)$. Al comparar las medias obtenidas por las familias de Educación Infantil $(\bar{x}=4,17$; $\mathrm{DT}=0,577)$ o las de Educación Primaria $(\bar{x}=4,22 ; \mathrm{DT}=0,561)$ con las de Secundaria $(\bar{x}=4,01$; $D T=0,705)$, las diferencias son significativas $(p<, 000)$, aunque el tamaño del efecto es también pequeño ( $d$ de Cohen 0,248 y 0,329, respectivamente, inferior a $d=0,5$ ), por lo que no procede considerarlo.

Junto a esta perspectiva de las familias cotejamos a la información proporcionada por los docentes como facilitadores o promotores de esa implicación en la educación escolar desde el hogar, no hemos encontrado diferencias significativas al contemplar la titularidad del centro $(p=, 431)$, ni la variable etapa educativa $(p=, 053)$, la situación laboral del profesorado $(p=, 443)$ o los años de experiencia $(p=, 694)$. En ocasiones en investigación, educativa en este caso, la no existencia de diferencias significativas, también es relevante.

\section{Discusión y conclusiones}

A partir del análisis de resultados previo, queda manifiesto que las familias valoran muy positivamente su implicación en la educación escolar de sus hijos e hijas desde el hogar. Son varios los trabajos que destacan la importancia de este hecho. Así Fernández-Hawrylak y Heras (2019) señalan que los resultados académicos de los niños mejoran cuando la implicación de las familias en la escuela es mayor. Cuando la escuela pone en marcha estrategias de colaboración, atendiendo a las particularidades de las familias, los niños estarán más influidos social y escolarmente, al tiempo que mejorará su rendimiento académico. En la misma línea, Santos et al. (2018), constatan que la implicación familiar produce una mejora del rendimiento escolar.

Esta implicación, en nuestro estudio, se concreta en aspectos como conocer que sus hijos e hijas asisten a clase, manifestar interés por lo que hacen en casa y el modo en que organizan su tiempo para dar respuesta a las tareas escolares, los refuerzos positivos que ofrecen a sus descendientes y la buena disposición para ayudarles. Según Núñez et al. (2012), el seguimiento de las tareas escolares por parte de la familia es de gran ayuda para la autorregulación y la creación de rutinas y hábitos de estudio en el alumnado. De igual modo Etxeberria e Intxausti (2013) y Lawson (2003), coinciden en señalar que esta implicación no se ciñe a la ayuda para resolver las cuestiones meramente académicas. En esta línea, coincidimos con Miranda y Castillo (2018), cuando realizan un estudio metodológico sobre el constructo apoyo y participación de la familia en los procesos educativos, determinan que el apoyo y la supervisión del aprendizaje en el hogar es una variable importante para la configuración de dicho constructo. Otras variables que complementan a la anterior, en la formación de ese constructo son comunicación con la escuela y conocimiento de la escuela.

Por otra parte, en relación a los hábitos lectores de las familias o con la asistencia a actividades culturales (cine, teatro, museos, viajes...) en el tiempo libre, observamos que la implicación familiar es algo menor. Esto puede conllevar a que los estudiantes estén menos motivados por las tareas lectoras y rindan menos en ellas. Consideramos que los hábitos lectores, en concreto, es una necesidad a tener en cuenta para fomentar su desarrollo. De este modo, estudios como el de Izquierdo, Sánchez y López (2019) y el de Mora-Figueroa, Galán y López-Jurado (2016), determinan que la implicación familiar influye en el hábito lector. Más concretamente, los estudiantes confirman que la preocupación de la familia por 
su trabajo en el colegio preguntándoles diariamente acerca del mismo, les ha influido positivamente en su hábito lector. En nuestro estudio, en contraste con los mencionados autores, la preocupación por las familias sobre lo que sus hijos e hijas trabajan a diario en el centro, se ha manifestado alta. Un hábito lector no del todo notable esté posiblemente condicionado por el hecho de que gran número de familias son migrantes y no saben leer y escribir en su idioma materno. También tienen la limitación idiomática de los recursos que se le ofrecen. Pero otra posible justificación, se podría encontrar en el incremento en volumen de tareas escolares experimentado en los niños de Educación Primaria, del 36\% al 64\%, en los últimos veinte años (hasta el 2002), según afirma Kohn (2013) puede estar restando tiempo a la familia en el hogar para que lo niños de edades tempranas realicen otras actividades tales como leer, jugar o asistencia a actividades culturales.

Al contrastar las opiniones que docentes y familias tienen acerca de la facilitación que el centro promueve para que la familia se implique más desde el hogar, encontramos que a mayor facilitación por parte del centro, más alta es la implicación desde el hogar. Esta percepción tiene un sentido bidireccional, tanto docentes como familias la comparten. Así, nuestros resultados coinciden con los aportados por otros autores (Andrés y Giró, 2016; Etxeberria e Intxausti, 2013), cuando señalan que los centros escolares y los docentes deben convertirse en los principales facilitadores de la implicación de las familias en los aprendizajes realizados desde el hogar. En esta línea, estudios como el de Pàmies-Rovira, Senent-Sánchez y Essomba-Gelabert (2016) señalan como principales factores de éxito en escuelas en contextos vulnerables el liderazgo pedagógico del equipo directivo, así como la implicación de todo el claustro de profesorado. Esto nos refuerza a impulsar que la facilitación de la implicación de las familias desde el hogar ha de venir promovida por los tutores, en primer lugar, el equipo docente y apoyada por el centro en general.

Respecto a la influencia que determinadas variables sociodemográficas (como origen nacional, titularidad del centro, etapa educativa, situación laboral del docente, experiencia docente) podían ejercer sobre la implicación de las familias en las tareas realizadas en el hogar, el origen nacional también influye, aunque sigue mostrando niveles altos de implicación (las familias migrantes entre algo y bastante, y las familias españolas entre bastante y mucho); y, por otra parte, la etapa educativa encontramos que esta determina variaciones en cuanto a la implicación de las familias. En este sentido, coincide con los resultados de Pérez-Díaz, Rodríguez y Sánchez (2001), indicando que normalmente el alumnado recibe ayuda de algún familiar: el $43 \%$ de las familias consideran que casi siempre o siempre ayudan a su hijos e hijas, y el $14 \%$ que los ayudan bastantes veces; estos porcentajes disminuyen en Educación Secundaria a un 9\%, observando que la implicación familiar se reduce conforme aumenta la edad. Nos encontramos ante el reto, tanto para el profesorado como a nivel de centro y comunidad de impulsar la implicación desde el hogar de las familias, manteniéndola estable hasta la educación secundaria, donde se hace más necesario este apoyo y seguimiento, dado el potencial educativo que puede desarrollar.

\section{Referencias}

Álvarez Blanco, L. (2019). Modelos teóricos de implicación educativa familiar: responsabilidades compartidas entre centros educativos, familias y comunidad. Aula Abierta, 48(1), 19-30. DOI: https://doi.org/10.17811/rifie.48.1.2019.19-30

Anderson, K. J., y Minke, K. M. (2007). Parent involvement in education: toward an understanding of parents' decision making. The Journal of Educational Research, 100(5), 311-323. DOI: 10.3200/JOER.100.5.311-323 
Andrés, S. y Giró, J. (2016). El papel y la representación del profesorado en la participación de las familias en la escuela. Revista Electrónica Interuniversitaria de Formación del Profesorado, 19(1), 61-71. DOI: http://dx.doi.org/10.6018/reifop.19.1.245461

Bolívar, A. (2006). Familia y escuela: dos mundos llamados a trabajar en común. Revista de Educación, 339, 119-146.

Bonell, L. (2016). La promoción de la participación educativa de las familias en la escuela desde una perspectiva transformadora: un estudio de casos. Tesis doctoral. Universidad de Valladolid.

Castro, M., Expósito, E., Lizasoain, L., López, E., y Navarro, E. (2014). Participación familiar y rendimiento académico. Una síntesis meta-analítica. En Consejo Escolar del Estado (Coord.) La participación de las familias en la educación escolar (pp. 83-105). Madrid: Ministerio de Educación, Cultura y Deporte.

Cohen, J. (1988) (2 $2^{\mathrm{a}}$ ed.). Statistical Power Analysis for the Behavioral Sciences. New York: Academic Press.

Chen, W-B. y Gregory, A. (2010). Parental involvement as a protective factor during the transition to high school. The Journal of Educational Research, 103(1), 53-62.

Dauber, S. L. y Epstein, J. L. (1989). Parent attitudes and practices of parent involvement in inner-city elementary and middle schools. Baltimore, Maryland: Center for Research on Elementary and Middle Schools.

Epstein, J.L. (2009). School, family, and community partnerships: caring for the children we share. En J. L. Epstein, M. G. Sanders, B. S. Simon, K. Clark, N. Rodriguez y F. L. Van Voorhis (Coords.), School, family, and community partnerships. Your handbook for action. Third Edition (pp. 7-29). London: Sage Publications.

Epstein, J. L. y Sheldon, S. B. (2002). Present and accounted for: improving student attendance through family and community involvement. Journal of Educational Research, 95(5), 308-318.

Etxeberria, F. e Intxausti, N. (2013). La percepción de los tutores sobre la implicación educativa de las familias inmigrantes. REOP, Revista Española de Orientación y Psicopedagogía, 24(3), 3. ${ }^{\text {er }}$ cuat., 43-62.

Fan, X. y Chen, M. (2001). Parental Involvement and Students' Academic Achievement: A meta analysis. Educational Psychology Review, vol. 13, núm. 1, 1-22.

Fernández-Freire, L., Rodríguez-Ruiz, B. y Martínez-González, R.A. (2019). Padres y madres ante las tareas escolares: la visión del profesorado. Aula Abierta, 48(1), 77-84. DOI: https://doi.org/10.17811/rifie.48.1.2019.77-84.

Fernández-Hawrylak, M. y Heras, D. (2019). Familias transnacionales, familias inmigrantes: Reflexiones sobre su inclusión en la escuela. Revista de Sociología de la Educación (RASE), 12, (1), 24-39. doi: http://dx.doi.org/10.7203/RASE.12.1.12787

Flecha, R. y Soler, M. (2013). Turning difficulties into possibilities: Engaging Roma families and students in school through dialogic learning. Cambridge Journal of Education, 43(4), 451-465.

Forest, C. y García-Bacete, F.J. (2006). Comunicación cooperativa entre la familia y la escuela. Valencia: Nau Llibres. 
García, R. (2016). Deberes escolares y rendimiento académico. Jaén: Universidad de Jaén.

García-Sanz, M.P. (2012). Fundamentos teóricos y metodológicos de la evaluación de programas. Murcia: DM.

Hernández-Prados, M.A., Gomariz Vicente, M.A. Parra Martínez, J. y García-Sanz, M.P. (2016). Familia, inmigración y comunicación con el centro escolar: Un estudio comparativo. Educación XXI, 19(2), 127-151. Recuperado de http://revistas.uned.es/index.php/educacionXX1/article/view/16457

Garreta, J.(2008). Escuela, familia de origen inmigrante y participación. Revista de Educación, núm. 345, 133-155.

Gomariz Vicente, M.A., Hernández-Prados, M.A., García-Sanz, M.P. y Parra Martínez, J. (2017). Facilitación de la participación de las familias en la educación de los hijos. Cuestionario a docentes. Recuperado de http://hdl.handle.net/10201/53822

González-Pineda, J. (2003). Adaptabilidad y cohesión familiar, implicación parental en conductas autorregulatorias, autoconcepto del estudiante y rendimiento académico. Psicothema, 15(3).471-477.

Grant, B.K. y Ray, J.A. (2013). Home, Shool, and Community Collaboration. London: Sage.

Hernández-Prados, M.A., Gomariz Vicente, M.A., Parra Martínez, J. y García-Sanz, M.P. (2017). Valoración de la participación de las familias en la educación de los hijos. Cuestionario a familias. Recuperado de http://hdl.handle.net/10201/53821

Hoover-Dempsey, K. V., Walker, J. M. T., Sandler, H. M., Whetsel, D., Green, C. L., Wilkins, A. S. y Closson, K. (2005). Why do parents become involved? Research and findings and implications. The Elementary School Journal, 106(2), 105-130. DOI: 10.1086/499194

Hoover-Dempsey, K. V., Battiato, A. C., Walker, J. M. T., Reed, R. P., Dejong, J. M., \& Jones, K. P. (2001). Parental involvement in homework. Educational Psychologist, 36(3), 195209.

Izquierdo, T., Sánchez, M. y López, M.D. (2019). Determinantes familiares en el fomento del hábito lector del alumnado de Educación Primaria. Estudios sobre educación, 36, 157179. DOI: 10.15581/004.36.157-179

Kohn, A. (2013). El mito de los deberes: ¿Por qué son perjudiciales para el aprendizaje y la convivencia? Madrid: Kaleida.

Lawson, M.A. (2003). School-family relations in context. Parent and teacher perceptions of parent involvement. Urban Education, 38(1), 77-133. DOI:10.1177/0042085902238687

Ley Orgánica 2/2006, de 3 de mayo, de Educación (2006) (BOE de 4 de mayo).

López, G. R., Scribner, J. D. y Mahitivanichcha, K. (2001). Redefining parental involvement: Lessons from high-performing migrant-impacted schools. American Educational Research Journal, 38(2), 253-288.

Madrona, P. (2001). Actitudes, hábitos y motivaciones de los alum- nos ante las tareas escolares en Educación Primaria visto desde la perspectiva de los padres, los maestros y los alumnos. Salamanca: Ediciones Universidad de Salamanca. 
Martín, E., Rodríguez, V. y Marchesi, A. (2005). La opinión de los profesores sobre la convivencia en los centros. Madrid: Instituto de Evaluación y Asesoramiento Educativo (IDEA). Centro de Innovación Educativa (CIE-FUHEM).

Miranda, C. y Castillo, P. (2018). Estudio metodológico de las variables que componen el constructo de "apoyo y participación de la familia en los procesos educativos". Estudios Pedagógicos XLIV, $\mathrm{N}^{\circ}{ }^{1}, 115-133$.

Mora-Figueroa, J., Galán, A. y López-Jurado, M. (2016). Eficacia de un programa de implicación familiar en la lectura de alumnado de $10^{\circ}$ de Educación Primaria. Revista de Psicodidáctica, 21(2), 375-391 DOI: 10.1387/RevPsicodidact.14889

Núñez, J. C., Suárez, N., Fernández, M., Cerezo, R., Rodríguez, C., y Rosario, P. (2012). Tareas para casa, implicación familiar y rendimiento académico. Aula Abierta, 40(1), 73-84.

Parra Martínez, J., Gomariz Vicente, M.A., Hernández-Prados, M.A. y García-Sanz, M.P. (2017). La participación de las familias en educación infantil. RELIEVE, 23(1), art. 4. DOI: http://doi.org/10.7203/relieve.23.1.9258.

Parra Martínez, J., Sánchez-López,C. y Gomariz Vicente, M.A. (2011). : Análisis del contexto familiar en educación. Revista Interuniversitaria de Formación del profesorado (digital), 14(1) 177-192.

Pàmies-Rovira, J., Senent-Sánchez, J.M. y Essomba-Gelabert, M.A. (2016). El liderazgo pedagógico y la implicación del profesorado como factores de éxito en centros de entornos desfavorecidos en España. RELIEVE, 22(2), art. 2. DOI: http://dx.doi.org/10.7203/relieve.22.2.7600

Pérez-Díaz, V., Rodríguez, J.C. y Sánchez, L. (2001). La familia española ante la educación de sus hijos. Barcelona: Fundación La Caixa.

Santos, M.A., Ferraces, M.J., Godas, A. y Lorenzo, M.M. (2018). Do cooperative learning and family involvement improve variables linked to academic performance? Psicothema, 30 (2), 212-217. DOI: 10.7334/psicothema2017.31

Valls-Caroll, R., Prados-Gallardo, M. y Aguilera-Jiménez, A. (2014). El proyecto INCLUD-ED: estrategias para la inclusión y la cohesión social en Europa desde la educación. Investigación en la escuela, 82, 31-43. DOI: http://dx.doi.org/10.12795/IE.2014.i82.03 